Systematic review

\title{
Effect of total-body prehabilitation on postoperative outcomes: a systematic review and meta-analysis
}

\author{
D. Santa Mina ${ }^{\mathrm{a}, \mathrm{b}, \mathrm{c}, *}$, H. Clarke ${ }^{\mathrm{d}, \mathrm{e}}$, P. Ritvo ${ }^{\text {b,f }}$, Y.W. Leung ${ }^{\mathrm{d}, \mathrm{e}}$, A.G. Matthew $^{\mathrm{a}, \mathrm{d}}$, \\ J. Katz ${ }^{\text {c,d,e }}$, J. Trachtenberg ${ }^{\text {a,d }}$, S.M.H. Alibhai ${ }^{\mathrm{d}, \mathrm{e}}$ \\ a Department of Surgical Oncology, Princess Margaret Cancer Center, Toronto, Ontario, Canada \\ ${ }^{\mathrm{b}}$ Kinesiology Program, University of Guelph-Humber, Toronto, Ontario, Canada \\ c School of Kinesiology and Health Science, York University, Toronto, Ontario, Canada \\ ${ }^{\mathrm{d}}$ University of Toronto, Toronto, Ontario, Canada \\ e University Health Network, Toronto, Ontario, Canada \\ ${ }^{\mathrm{f}}$ Cancer Care Ontario, Toronto, Ontario, Canada
}

\begin{abstract}
Objective To systematically review the evidence of pre-operative exercise, known as 'prehabilitation', on peri- and postoperative outcomes in adult surgical populations.

Design Systematic review and meta-analysis.

Data sources CENTRAL, Medline, EMBASE, CINAHL, PsycINFO and PEDro were searched from 1950 to 2011.

Methods Two reviewers independently examined relevant, English-language articles that examined the effects of pre-operative total-body exercise with peri- and postoperative outcome analysis. Given the nascence of this field, controlled and uncontrolled trials were included. Risk of bias was assessed using the Cochrane Risk of Bias Assessment tool. Only data on length of stay were considered eligible for meta-analysis due to the heterogeneity of measures and methodologies for assessing other outcomes.

Results In total, 4597 citations were identified by the search s trategy, of which 21 s tudies w ere included. Trials w ere generally small (median $=54$ participants) and of moderate to poor methodological quality. Compared with standard care, the majority of studies found that total-body prehabilitation improved postoperative pain, length of stay and physical function, but it was not consistently effective in improving health-related quality of life or aerobic fitness in the studies that examined these outcomes. The meta-analysis indicated that prehabilitation reduced postoperative length of stay with a small to moderate effect size (Hedges' $g=-0.39, P=0.033$ ). Intervention-related adverse events were reported in two of 669 exercising participants.

Conclusion The literature provides early evidence that prehabilitation may reduce length of stay and possibly provide postoperative physical benefits. Cautious interpretation of these findings is warranted given modest methodological quality and significant risk of bias.
\end{abstract}

Exercise; General surgery; Preoperative care; Preoperative period; Rehabilitation; Postoperative period

Keywords: Pre-operative exercise; Prehabilitation; Postoperative outcomes; Systematic review; Meta-analysis

\section{Introduction}

The acute postoperative period is associated with a marked reduction in physical function and health-related

\footnotetext{
* Corresponding author at: The University of Guelph-Humber, Room GH-308-G, 207 Humber College Boulevard, Toronto, Ontario M9W 5L7, Canada. Tel.: +1 416798 1331x6065; fax: +1 4167983293 .

E-mail addresses: Daniel.santamina@guelphhumber.ca, dstamina@gmail.com (D. Santa Mina).
}

quality of life (HRQOL) [1]. Pre-operative physical conditioning is an increasingly common strategy aimed at improving postoperative outcomes, including length of stay (LOS), functional capacity and peri-operative complications [2-5]. Furthermore, the typical waiting period may represent an ideal time to invest in chronic health with a general exercise programme, ostensibly capitalising on the psychological impact ('teachable moment') of recent diagnosis to facilitate changes in health behaviour that include regular physical activity. This strategy, known as 'prehabilitation', 
commonly employs physiotherapy modalities or more common exercises targeting specific muscles or joints, often without targeting the systemic musculoskeletal and cardiovascular deconditioning that follows prolonged immobilisation. This focused approach ignores evidence from multiple studies, demonstrating that pre-operative systemic physical fitness positively predicts peri-operative complications and functional recovery, that have clinical and economic salience [6,7]. Consequently, a more focused examination of trials that employ broader fitness enhancement strategies is needed to determine whether total-body exercise can improve the surgical experience and recovery. While recent reviews have described a net benefit of prehabilitation [3-5], they have not conducted a meta-analysis nor have they focused specifically on total-body prehabilitation strategies across surgical populations. Therefore, the purpose of this study was to systematically review and meta-analyse the current literature examining the effect of total-body prehabilitation interventions on peri- and postoperative outcomes in adults undergoing surgical intervention.

\section{Methods}

\section{Search strategy}

Studies published in English between 1950 and August 2011 were recovered from the following databases by an experienced medical information specialist: the Cochrane Central Register of Controlled Trials (CENTRAL), MEDLINE, EMBASE, CINAHL, PsycINFO and PEDro. Search terms are presented in Appendix A (see online supplementary material). Hand searches of the reference lists of included studies were conducted to identify any additional relevant trials. Fig. A (see online supplementary material) shows a summary of the selection process. The corresponding authors of included trials were contacted to identify any potential additional relevant trials.

Two reviewers independently reviewed abstracts identified by the search for potentially relevant trials. Full-text articles of relevant studies were obtained and reviewed to confirm inclusion. Discrepancies between reviewers regarding inclusion were resolved by a third reviewer.

\section{Inclusion criteria}

Eligible trials included prospective, prehabilitation interventions that evaluated the effect of pre-operative total-body exercise for patients aged $\geq 18$ years undergoing curative or palliative surgery. Exercise was operationally defined as non-site-specific structured physical activity that included cardiovascular and/or resistance training of the upper and/or lower extremities. Studies were included when the intervention(s) included exercise prescriptions with an indication of frequency (sessions/week), intensity (e.g. percentage maximum heart rate, rating of perceived exertion, etc.), time (duration of exercise session in minutes) and/or type of exercise (e.g. walking, cycling, resistance training). There was no limitation to intervention duration or intensity. Studies for inclusion required both pre- and peri- or postoperative outcome measurements; given the nascence of this field, there was no exclusion based on study design.

\section{Data extraction}

Two reviewers independently evaluated all studies for content and methodological quality using a standardised data extraction table. The reviewers were blinded to each other's abstraction process but were not blinded to each study's authors, date, journal or title. Study outcomes were grouped according to the following categories: HRQOL, pain, musculoskeletal and functional task performance, aerobic fitness, postoperative LOS and healthcare utilisation, peri-operative complications and adverse events. Outcome statistical significance was set to $P \leq 0.05$ and intention-totreat results were used when available. The corresponding authors of included studies were contacted by email to verify the methodology and results.

\section{Risk of bias assessment}

Study risk of bias was evaluated according to the Cochrane Risk of Bias Assessment tool [8] (Table 1). A third reviewer resolved any disagreements regarding classification of study quality components.

\section{Meta-analysis}

A meta-analysis was performed on outcomes that were used consistently by three or more studies using Comprehensive Meta-Analysis, Version 2 [9]. Effect size was calculated as Hedge's $g$, which denotes the mean difference between the intervention and control groups divided by the pooled standard deviation of the two groups. To aggregate effect sizes, each effect size was weighted by the inverse of its variance, while the weighted effect sizes were summed across studies and then divided by the sum of the weights.

Cochrane's $Q$ test and the $I^{2}$ statistic were used to assess heterogeneity between studies [10]. Publication bias was assessed by conducting a funnel plot, the Begg and Mazumdar rank correlation test [11] and Egger's regression asymmetry test [12].

\section{Results}

\section{Search results}

The database search yielded 4597 citations, plus an additional 41 studies identified through hand searches and communication with authors. In total, 89 candidate studies were examined in detail, of which 22 studies met the inclusion criteria [6,13-33] (see Fig. A, online supplementary 
Table 1

Cochrane risk of bias assessment.

\begin{tabular}{|c|c|c|c|c|c|c|c|}
\hline Study & $\begin{array}{l}\text { Adequate } \\
\text { sequence } \\
\text { generation? }\end{array}$ & $\begin{array}{l}\text { Allocation } \\
\text { concealment? }\end{array}$ & Blinding? & $\begin{array}{l}\text { Incomplete } \\
\text { outcome data } \\
\text { addressed? }\end{array}$ & $\begin{array}{l}\text { Free of } \\
\text { selective } \\
\text { reporting? }\end{array}$ & $\begin{array}{l}\text { Free of other } \\
\text { bias? }\end{array}$ & $\begin{array}{l}\text { Total } \\
\text { score } / 6\end{array}$ \\
\hline Arthur et al. [13] & $\mathrm{Y}$ & $\mathrm{Y}$ & $\mathrm{Y}$ & $\mathrm{Y}$ & $\mathrm{N}$ & ?c & 4 \\
\hline Back et al. [14] & $\mathrm{Y}$ & $\mathrm{N}$ & $\mathrm{N}$ & $? \mathrm{~b}$ & $\mathrm{Y}$ & $\mathrm{Y}$ & 3 \\
\hline Carli et al. [25] & $\mathrm{Y}$ & $?$ & $\mathrm{~N}$ & $\mathrm{Y}$ & $\mathrm{Y}$ & $\mathrm{Y}$ & 4 \\
\hline D’Lima et al. [15] & $\mathrm{Y}$ & $\mathrm{N}$ & $?$ & ?b & $?$ & $? \mathrm{c}$ & 1 \\
\hline Dronkers et al. [26] & $? \mathrm{a}$ & $\mathrm{Y}$ & $\mathrm{Y}$ & $? \mathrm{~b}$ & $\mathrm{Y}$ & $\mathrm{Y}$ & 4 \\
\hline Evgeniadis et al. [16] & $\mathrm{Y}$ & $\mathrm{Y}$ & $\mathrm{Y}$ & ?b & $?$ & $? \mathrm{c}$ & 3 \\
\hline Ferrara et al. [33] & $\mathrm{Y}$ & $?$ & $\mathrm{Y}$ & ?b & $?$ & $\mathrm{Y}$ & 3 \\
\hline Gilbey et al. [17] & $\mathrm{Y}$ & $?$ & $?$ & $? \mathrm{~b}$ & $?$ & $? \mathrm{c}$ & 1 \\
\hline Gocen et al. [18] & $\mathrm{Y}$ & $\mathrm{N}$ & $\mathrm{Y}$ & $? \mathrm{~b}$ & $\mathrm{Y}$ & $? \mathrm{~d}$ & 3 \\
\hline Herdy et al. [22] & $\mathrm{Y}$ & $?$ & $?$ & $? \mathrm{~b}$ & $\mathrm{Y}$ & $? \mathrm{c}$ & 2 \\
\hline Hoogeboom et al. [27] & $? \mathrm{a}$ & $\mathrm{Y}$ & $\mathrm{Y}$ & $? \mathrm{~b}$ & $?$ & $\mathrm{Y}$ & 3 \\
\hline Jaggers et al. [19] & $\mathrm{N}$ & $\mathrm{N}$ & $\mathrm{N}$ & NA & $?$ & $\mathrm{~N}$ & 0 \\
\hline $\begin{array}{l}\text { Jones } \text { et al. }[6] \text { and } \\
\text { Peddle } \text { et al. }[21]\end{array}$ & $\mathrm{N}$ & NA & $\mathrm{Y}$ & $? \mathrm{~b}$ & $\mathrm{Y}$ & $\mathrm{N}$ & 2 \\
\hline Nielsen et al. [20] & Y & $\mathrm{Y}$ & $\mathrm{N}$ & ?b & $?$ & $\mathrm{Y}$ & 3 \\
\hline Rodgers et al. [32] & $\mathrm{N}$ & $\mathrm{N}$ & $\mathrm{N}$ & ?b & $?$ & $? \mathrm{c}$ & 0 \\
\hline Rooks et al. [23] & $\mathrm{Y}$ & $?$ & $?$ & ?b & $?$ & $? \mathrm{c}$ & 1 \\
\hline Rosenfeldt et al. [28] & Y & $?$ & $?$ & ?b & $\mathrm{Y}$ & $? \mathrm{c}$ & 2 \\
\hline Sekine et al. [29] & $\mathrm{N}$ & NA & $?$ & ?b & $?$ & ?e & 0 \\
\hline Topp et al. [24] & $\mathrm{Y}$ & $?$ & $\mathrm{Y}$ & $? \mathrm{~b}$ & $?$ & $\mathrm{Y}$ & 3 \\
\hline Wang et al. [30] & $\mathrm{Y}$ & $?$ & $\mathrm{~N}$ & $? \mathrm{~b}$ & $?$ & $\mathrm{Y}$ & 2 \\
\hline Williamson et al. [31] & $\mathrm{Y}$ & $\mathrm{Y}$ & $\mathrm{Y}$ & $\mathrm{Y}$ & $?$ & $\mathrm{Y}$ & 5 \\
\hline
\end{tabular}

Y, yes; N, no; NA, not applicable; ?, insufficient or inadequate information; ?a, used 'prepared' or 'sealed' envelopes (method of creating random assignment, e.g. 'shuffled' or 'mixed', not stated); ?b, approach to volume and approach to resolving missing data was not reported; ?c, no intention-to-treat analysis for postoperative outcomes; ?d, baseline differences in age (46.9 vs $55.5 ; P=0.01)$ and body mass index $(24.9$ vs $27.7 ; P=0.06)$; ?e, numerous elements of methods were not reported.

material). More detailed review revealed that two studies described different outcomes for the same cohort, and were included as one trial in this review $[6,21]$. Nine of the candidate studies required arbitration from the third reviewer. In total, 21 studies were included in this review.

\section{Risk of bias}

Within-study risk of bias is presented in Table 1. Out of a maximum score of 6 (higher scores indicate lower risk of bias), one study achieved a score of 6 [31], three studies achieved a score of $4[13,25,26]$, seven studies achieved a score of 3 [14,16,18,20,24,27,33], four studies achieved a score of $2[21,40]$, three studies achieved a score of $1[15,17,23]$, and three studies achieved a score of 0 $[19,29,32]$.

\section{Study characteristics}

Included studies are summarised in Table 2. Studies were published between 1996 and 2011. Of the 21 included trials, 17 were randomised controlled trials (RCTs) [13-18,20,22-28,30,31,33], with one non-RCT [32], one single-group trial (with secondary outcomes published separately) [6,21], one prospective case-control study using historical control subjects [29], and one randomised casestudy design (two participants, each randomised to either prehabilitation or usual care) [19]. In total, 1371 participants were included in these 21 trials. Thirteen studies assessed pre-operative exercise in orthopaedic populations [15,16,18-20,23,24,27,30-34], and eight studies examined pre-operative exercise in patients undergoing visceral organ surgery $[6,13,14,21,22,25,26,28,29]$.

\section{Intervention characteristics}

Six trials examined supervised, facility-based interventions [6,13,15,21-23,26,27,31,32], four were home-based (with or without periodic direct supervision from study staff) $[14,16,20,25]$, and four used a combination of facility-based and home-based exercises [17,19,24,28-30,33]. Aerobic exercise was the primary exercise modality in five studies [6,13,21,27-29], resistance or calisthenic exercise in two studies [16,18], a mixed-modality approach (aerobic plus resistance) was used in thirteen studies [14,17,19,20,22-26,30-33], and one study compared aerobic exercise with resistance exercise [15]. The median intervention duration was 6 weeks (range 1 to 8 weeks). To complement the pre-operative exercise, several studies included ancillary intervention components, such as patient education relevant to the surgical and recovery process $[13,18,20,33]$ and dietary provision [20].

\section{Effectiveness of pre-operative exercise interventions}

\section{Pre-operative, postintervention outcomes}

Pre-operative, postintervention measurements were conducted in 17 trials and occurred between 1 and 7 days 
Table 2

Study characteristics.

\begin{tabular}{lll}
\hline Study & Design and sample & Intervention \\
\hline D'Lima et al. [15] & Design: three-arm RCT & Intervention design/setting: supervised, facility-based exercise \\
& $N=30(\mathrm{TX} 1: n=10 ; \mathrm{TX} 2: n=10 ; \mathrm{CON}: n=10)$ & F: 3 sessions/week; I: see Exercise specifics below; D: 45 \\
Sample characteristics: osteoarthritis or rheumatoid & minutes/session; L: 6 weeks \\
arthritis with scheduled total knee replacement & TX1: Musculoskeletal physical therapy \\
Mean age (SD) in years: TX1 =68.5 (4.6); TX2 $=71.6$ & Exercise specifics: flexibility training of calf, hamstring and \\
& quadriceps. Isotonic and isometric strength training of triceps \\
& surae, quadriceps, hamstrings, hip flexors, hip extensors, hip \\
& abductors, shoulder flexors, shoulder abductors and triceps \\
& brachii. Weights used as tolerated \\
& TX2: Cardiovascular conditioning \\
& I: 40 to 70\% of heart rate reserve \\
& Exercise specifics: exercises included arm or cycle ergometry or \\
& aquatic exercises
\end{tabular}

Evgeniadis et al. [16] Design: three-arm RCT

$N=53$ (TX1: $n=18$; TX2: $n=15 ; \mathrm{CON}: n=20$ )

Sample characteristics: idiopathic knee osteoarthritis

Mean age (SD) in years: TX1 $=67.1$ (4.4); TX2 $=68.6$

(5.9); $\mathrm{CON}=69.4(1.9)$

Ferrara et al. [33]

Design: RCT

$N=23$ (TX: $n=11 ;$ CON: $n=12$ )

Sample characteristics: end-stage osteoarthritis

scheduled for total hip arthroplasty

Mean age (SD) in years: $\mathrm{TX}=63.8(9.0) ; \mathrm{CON}=63.1$

(6.9)

Gilbey et al. [17]

Design: RCT

$N=57$ (TX: $n=32 ;$ CON: $n=25$ )

Sample characteristics: hip joint disease (osteoarthritis, post-traumatic arthritis, inflammatory arthritis, osteonecrosis or Paget's disease) with indication for total hip arthroplasty

Mean age (SD) in years: $\mathrm{TX}=66.7(10.2) ; \mathrm{CON}=63.3$ (12.0)

Intervention design/setting: supervised, home-based

TX1: Pre-operative exercise

F: 3 sessions/week; I: 3 sets of 10 to 14 reps (progressive); D:

NR; L: 3 weeks

Exercise specifics: each session had warm-up, RET, cool-down. RET exercises using resistance bands targeted the upper back, shoulders, humeral bicep, triceps, deltoid, rectus abdominus and femoral flexors

TX2: Postoperative exercise

L: 8 weeks

Exercise specifics: programme was similarly structured to TX1. Exercises were designed to strengthen posterior tibial muscles, anterior and posterior femoral muscle and femoral abductors

Intervention design/setting: supervised, facility-based group (40 minutes) and individual (20 minutes) exercise

F: 5 days/week; I: NR; D: 60 minutes; L: 4 weeks

Exercise specifics:

RET: leg stretching, hip abductor and quadriceps strengthening for 3 to 4 sets of 8 to 12 reps

AET: recumbent cycling for 10 to 15 minutes at low to moderate intensity

Ancillary intervention components [treatment arm (s) only]: education for preventing dislocation of prostheses, use of assistive devices, correct posture and activities of daily living. Postural therapy, including re-alignment of the spine, shoulders, elbows, wrists, hands, hips, knee and ankles

Design/setting: facility- and home-based exercise F: 4 days/week; I: NR; D: 60 minutes; L: 8 weeks Exercise specifics:

Facility-based exercise: 30 minutes of AET and RET, followed by 30 minutes of mobility and gait training in the hydrotherapy pool

RET: heel raise, leg flexion/extension, thigh flexion/extension, isometric thigh abduction, trunk flexion, trunk rotation, forearm curls, seated body raise and hip hikes; 1 to 3 sets of 10 reps for isotonic exercises; isometric exercises $=2$ sets of 10 repetitions with 5-s holds

Gocen et al. [18] Design: RCT

$N=59$ (TX: $n=29$; CON: $n=30$ )

Sample characteristics: hip osteoarthritis

Mean age (SD) in years: TX $=46.93$ (11.48);

$\mathrm{CON}=55.5$ (14.44) (between-group difference $P<0.01$ )

Intervention design/setting: NR

F: 7 days/week; 3 sessions/day; I: 10 reps; D: NR; L: 8 weeks Exercise specifics: straight leg raises, hamstring and hip flexor stretches, and strengthening of upper extremities

Ancillary intervention components [treatment arm (s) only]: education programme regarding movements that should be avoided, posture, lifting, carrying, bathing and use of assistive devices 
Table 2 (Continued)

\begin{tabular}{lll}
\hline Study & Design and sample & Intervention \\
\hline Hoogeboom et al. [27] & Design: RCT & Intervention design/setting: outpatient physiotherapy department \\
& $N=21$ (TX: $n=10 ; \mathrm{CON}: n=11)$ & F: minimum of 2 days/week; I: 13 to 14/20 RPE; D: 60 minutes; \\
& Sample characteristics: hip osteoarthritis & L: 3 to 6 weeks \\
& Mean age (SD) in years: TX=77 (3); CON=75 (5) & Exercise specifics: 5 minutes of warm-up (walking), leg press, 30 \\
& minutes of stationary cycling, functional training for activities of \\
& daily living \\
& Ancillary intervention components [treatment arm (s) only]: \\
& encouraged to exercise at home as well and provided with a \\
& pedometer
\end{tabular}

Jaggers et al. [19] Design: RCT

$N=2$ (TX: $n=1 ;$ CON: $n=1)$

Sample characteristics: female, osteoarthritis of right knee

Age in years: $\mathrm{TX}=62 ; \mathrm{CON}=57$

Nielsen et al. [20]

Rodgers et al. [32]

Rooks et al. [23]

Topp et al. [24]
Design: RCT

$N=60$ (TX: $n=28 ;$ CON: $n=32$ )

Sample characteristics: degenerative disease of low Mean age (range) in years: TX $=48$ (31 to 80 ); $\mathrm{CON}=52(23$ to 88$)$

Design: controlled trial (assignment to TX and CON based on geographical location)

$N=20$ (TX: $n=10 ;$ CON: $n=10$ )

Sample characteristics: knee osteoarthritis

Mean age (SD) in years: $\mathrm{TX}=70(63$ to 78$) ; \mathrm{CON}=65$ (50 to 83 )

Design: RCT (CON underwent education intervention) $N=78$ (TX: $n=39$; CON: $n=39$ )

Sample characteristics: advanced hip or knee osteoarthritis Mean age (SD) in years: TX (hip) $=65(11)$; CON $($ hip $)=59(7) ; \mathrm{TX}(\mathrm{knee})=65(8) ; \mathrm{CON}(\mathrm{knee})=69(8)$

Design: RCT

$N=54$ (TX: $n=26 ;$ CON: $n=28$ )

Sample characteristics: knee osteoarthritis

Mean age (SD) in years: $\mathrm{TX}=64.1(7.05) ; \mathrm{CON}=63.5$ (6.68) back with low back and radiating pain
Intervention design/setting: supervised (once/week) and unsupervised (twice/week)

F: 3 times/week; L: 4 weeks

Exercise specifics: 10 minutes of warm-up (walking and stretching), 30 minutes of RET (nine lower-body exercises) using resistance bands, 10 minutes of step training and 5 minutes of cool-down (light static stretching)

Intervention design/setting: home-based exercise (instructed by physiotherapist at day of recruitment and 2 weeks pre-operatively) F: daily; I: NR; D: 30 minutes; L: 6 to 8 weeks

Exercise specifics: exercises focused on improvement of back and abdomen muscle strengthening and cardiovascular conditioning Ancillary intervention components [treatment arm (s) only]: education about the operation, postoperative mobilisation and rehabilitation; on the day before surgery, TX group was given $200 \mathrm{ml}$ of protein-rich drink. Postoperative rehabilitation in the TX group included intensive mobilisation on the day of surgery and 30 minutes twice daily for the following days with aim to discharge on fifth postoperative day

Intervention design/setting: supervised, facility-based F: 3 times/week; I: NR; D: NR; L: 6 weeks

Exercise specifics: stretching and warm-up, heel slides (supine knee flexion), isometric quadriceps sets, straight leg raises, short-arc quadriceps sets, standing squats, step-ups and cycling

Intervention design/setting: supervised, facility-based F: 3 times/week; I: moderate intensity; D: NR; L: 6 weeks Exercise specifics:

During first 3 weeks: participants performed 1 to 2 sets of 8 to 12 reps of single-joint movements while standing in chest-deep water, focusing on single planar motion of the cervical spine, shoulders, elbows, wrists, hands, hips, knees and ankles During last 3 weeks: participants performed moderate-intensity AET on a recumbent bicycle or elliptical machine, and RET (seated row, chest press, leg press, bicep curls and triceps kickbacks, as well as some calisthenic-type exercises). Two sets of 8 to 12 reps per exercise were prescribed At the end of each session, participants performed flexibility training

Intervention design/setting: mixed setting (twice/week unsupervised, home-based and once/week supervised, facility-based)

F: 3 times/week; I: NR; D: NR; L: minimum 4 weeks (mean number of sessions $=13$, range $=4$ to 23 )

Exercise specifics: each session had: 5-minute warm-up followed by nine lower-body RET using resistance bands (squats, hip flexion and extension, hip abduction and adduction, ankle plantar flexion and dorsiflexion, and knee extension and flexion); AET included lateral and vertical stepping on a $8^{\prime}$ step, finishing with a cool-down of light static stretching of gluteus, hip, hamstring, calf, torso, upper back, lower back and triceps and cooling down with 5 minutes of light walking 
Table 2 (Continued)

\begin{tabular}{|c|c|c|}
\hline Study & Design and sample & Intervention \\
\hline Wang et al. [30] & $\begin{array}{l}\text { Design: RCT } \\
N=28 \text { (TX: } n=15 ; \text { CON: } n=13) \\
\text { Sample characteristics: hip osteoarthritis }(n=25) \text {, } \\
\text { osteonecrosis }(n=2) \text { or inflammatory arthritis }(n=1) \\
\text { Mean age (SD) in years: TX }=68.3(8.2) \text { CON }=65.7 \\
(8.4)\end{array}$ & $\begin{array}{l}\text { Intervention design/setting: mixed setting (twice/week } \\
\text { unsupervised, home-based and twice/week supervised, } \\
\text { facility-based) } \\
\text { F: } 4 \text { times/week; I: participant-determined; D: } 60 \text { minutes; L: } 8 \\
\text { weeks } \\
\text { Exercise specifics: each session included hydrotherapy, stationary } \\
\text { cycling and resistance training (hip abduction/flexion/extension, } \\
\text { knee flexion/extension, ankle plantar flexion). Participants were } \\
\text { instructed to increase resistance by } 5 \mathrm{~kg} \text { when they could } \\
\text { comfortably do } 3 \text { sets of } 10 \text { reps } \\
\text { Ancillary intervention components [treatment arm(s) only]: } \\
\text { supervised clinic and home-based exercise, starting } 3 \text { weeks } \\
\text { postoperatively (after surgical wound was healed) }\end{array}$ \\
\hline
\end{tabular}

Williamson et al. [31] Design: RCT; three arms: pre-operative exercise (TX1) vs pre-operative acupuncture (TX2 vs $\mathrm{CON}$ ) $N=181(\mathrm{TX} 1: n=60 ;$ TX2: $n=60, \mathrm{CON}: n=61)$ Sample characteristics: patients on waiting list for total knee arthroplasty due to osteoarthritis Mean age $(\mathrm{SD})$ in years: $\mathrm{TX} 1=70.0(8.8) ; \mathrm{TX} 2=72.4$ (7.7); $\mathrm{CON}=69.6(10.0)$

Arthur et al. [13]

Design: RCT

$N=246$ (TX: $n=123 ; \mathrm{CON}: n=123$ )

Sample characteristics: low-risk patients awaiting elective coronary artery bypass graft with a minimum wait time of 10 weeks

Mean age (SD) in years: $\mathrm{TX}=61.8(8.4) ; \mathrm{CON}=63.8$ (7.8)

Back et al. [14]

Design: RCT

$N=37$ (TX: $n=21 ; \mathrm{CON}: n=16)$

Sample characteristics: stable coronary artery disease awaiting percutaneous coronary intervention Mean age (range) in years: $\mathrm{TX}=61.5$ (59.8 to 65.5); $\mathrm{CON}=64(58.5$ to 71$)$

Carli et al. [25]

Design: RCT

$N=112$ (TX: $n=58 ; \mathrm{CON}: n=54)$

Sample characteristics: colorectal surgery patients

Mean age (SD) in years: TX $=61(16) ; \mathrm{CON}=60(15)$

Intervention design/setting: facility-based, group exercise F: once/week; I: NR; D: 60 minutes; L: 6 weeks

Exercise specifics: circuit-based exercise class (repetitions and sets NR) consisting of: static quadriceps contractions, inner range quadriceps contractions, straight leg raises, sit to stands, stair climbing, calf stretches, resisted knee extensions, balance training, knee flexion and extension, freestanding pedal revolutions

TX2: Weekly lower extremity acupuncture for 6 weeks pre-operatively

Intervention design/setting: supervised, facility-based group exercise

F: twice/week; I: 40 to $70 \%$ of functional capacity; D: 90 minutes/session; L: 8 weeks

Exercise specifics: each session consisted of 5 to 10 minutes of warm-up, 10 minutes of stretching, a minimum of 30 minutes of interval AET, and 5 to 10 minutes of cool-down and stretching Ancillary intervention components [treatment arm (s) only]: educational intervention at baseline and 1 week pre-operatively

Intervention design/setting: unsupervised; home-based F: 5 days/week; I: see Exercise specifics below; D: see Exercise specifics below; L: 32 weeks (training initiated 8 weeks before percutaneous coronary intervention and continued postoperatively)

Exercise specifics: exercises performed on cycle ergometer with option to do alternative AET 2 days/week. Changes to AET intensity were based on monthly assessments. Resistance band exercises included: unilateral shoulder flexion, unilateral shoulder abduction, bilateral rowing and unilateral heel-lift (without bands) AET:

I: $70 \%$ of $\mathrm{VO}_{2}$ max or RPE of 13 to 15

D: 30 minutes (including 10-minute warm-up and 5-minute cool-down)

RET:

I: 3 sets of 10 repetitions at $75 \%$ of $1 \mathrm{RM}$

Intervention design/setting: home-based (equipment provided) F: 7 days/week (AET = 7 days/week; RET = 3 days/week); L: median pre-operative intervention duration was 38 days (interquartile range $=22$ to 60 )

Exercise specifics:

AET: I: stationary cycling at $50 \%$ of measured maximum heart rate, increased by $10 \%$ each week if tolerable; D: 20 to 30 minutes RET: I: push-ups, sit-ups and lunges until volitional fatigue; free-weight exercises for biceps, deltoids and quadriceps at 8RM; D: 10 to 15 minutes 
Table 2 (Continued)

\begin{tabular}{|c|c|c|}
\hline Study & Design and sample & Intervention \\
\hline Dronkers et al. [26] & $\begin{array}{l}\text { Design: RCT } \\
N=42 \text { (TX: } n=22 ; \mathrm{CON}: n=20) \\
\text { Sample characteristics: patients awaiting elective colon } \\
\text { surgery } \\
\text { Mean age (SD) in years: } \mathrm{TX}=71.1(6.3) ; \mathrm{CON}=68.8 \\
(6.4)\end{array}$ & $\begin{array}{l}\text { Intervention design/setting: outpatient physiotherapy department } \\
\text { F: } 2 \text { days/week; D: } 60 \text { minutes; L: } 2 \text { to } 4 \text { weeks } \\
\text { Exercise specifics: } \\
\text { AET: I: } 55 \text { to } 75 \% \text { of maximal heart rate; } 10 \text { to 13/20 RPE; D: } 20 \\
\text { to } 30 \text { minutes } \\
\text { RET: lower limb extensors, } 1 \text { set of } 8 \text { to } 15 \text { reps } \\
\text { Ancillary intervention components [treatment arm (s) only]: } \\
\text { inspiratory muscle training; encouraged to exercise at home as } \\
\text { well and provided with a pedometer }\end{array}$ \\
\hline Herdy et al. [22] & $\begin{array}{l}\text { Design: RCT } \\
N=56 \text { (TX: } n=29 ; \mathrm{CON}: n=27) \\
\text { Sample characteristics: } \\
\text { patients with acute coronary syndrome awaiting } \\
\text { coronary artery bypass graft } \\
\text { Mean age (SD) in years: } \mathrm{TX}=61(10) ; \mathrm{CON}=58(9)\end{array}$ & $\begin{array}{l}\text { Intervention design/setting: supervised, facility-based } \\
\text { F: NR; I: } 2 \text { to } 4 \text { METS; D: NR; L: minimum of } 5 \text { days } \\
\text { (mean = } 6.7 \text { days) } \\
\text { Exercise specifics: progressive exercises as per phase } 1 \text { cardiac } \\
\text { rehabilitation (light aerobic and musculoskeletal exercise focused } \\
\text { on activities of daily living) } \\
\text { Ancillary intervention components [treatment arm (s) only]: } \\
\text { postoperative rehabilitation following extubation }\end{array}$ \\
\hline $\begin{array}{l}\text { Jones } \text { et al. }[6] \text { and Peddle } \\
\text { et al. }[21] \\
\text { Same cohort from two } \\
\text { studies }\end{array}$ & $\begin{array}{l}\text { Design: prospective, single-group trial } \\
N=13 \text { (completed pre- and postsurgery assessment) } \\
\text { Sample characteristics:suspected Stage I to IIIA } \\
\text { non-small cell lung cancer } \\
\text { Mean age (SD) in years: } 65 \text { (10) }\end{array}$ & $\begin{array}{l}\text { Design/setting: supervised, facility-based } \\
\text { F: } 5 \text { times/week (consecutive days); I: } 60 \text { to } 65 \% \mathrm{VO}_{2} \text { peak; D: } 20 \\
\text { to } 30 \text { minutes; L: } 4 \text { to } 8 \text { weeks [mean time from diagnosis to } \\
\text { surgical resection }=67 \text { (7) days] } \\
\text { Exercise specifics: cycle ergometry at varying intensities: from } \\
\text { the second week onwards, participants integrated high-intensity } \\
\text { cycling (at ventilator threshold for } 20 \text { to } 25 \text { minutes). From the } \\
\text { fourth week onwards, participants integrated interval training ( } 6: 1 \\
\text { to } 4: 1 \text { ). Each session included a 5-minute warm-up and 5-minute } \\
\text { cool-down }\end{array}$ \\
\hline Rosenfeldt et al. [28] & $\begin{array}{l}\text { Design: RCT } \\
N=117 \text { (TX: } n=60 ; \text { CON: } n=57) \\
\text { Sample characteristics: elective coronary artery bypass } \\
\text { graft and/or cardiac valve surgery } \\
\text { Mean age (range) in years: TX }=62.5(59.0 \text { to } 68.5) \text {; } \\
\text { CON=68 (58.0 to 77.0) (between-group difference } \\
P=0.06)\end{array}$ & $\begin{array}{l}\text { Intervention design/setting: first } 2 \text { weeks, supervised, } \\
\text { facility-based; from } 2 \text { weeks to surgery, exercise was } \\
\text { unsupervised, home-based } \\
\text { F: } 4 \text { days/week for first } 2 \text { weeks ( } 2 \text { days in facility plus } 2 \text { days at } \\
\text { home); I: up to } 60 \% \text { of maximum heart rate; D: } 15 \text { to } 40 \text { minutes; } \\
\text { L: } 8 \text { weeks } \\
\text { Exercise specifics: AET circuit including cycle ergometry, } \\
\text { treadmill walking and arm ergometry. After the first } 2 \text { weeks, } \\
\text { participants were encouraged to continue exercising (walking) at } \\
\text { home for } 4 \text { days/week (provided with heart rate monitor for } \\
\text { intensity observation) } \\
\text { Ancillary intervention components [treatment arm (s) only]: four } \\
60 \text {-minute mental stress reduction therapy delivered by an } \\
\text { occupational therapist for first } 2 \text { weeks }\end{array}$ \\
\hline Sekine et al. [29] & $\begin{array}{l}\text { Design: case-control study (prospectively enrolled } \\
\text { participants into the intervention vs historical data from } \\
\text { patients that did not receive the intervention) } \\
N=82 \text { (TX: } n=22 ; \mathrm{CON}: n=60) \\
\text { Sample characteristics: patients with non-small-cell lung } \\
\text { carcinoma and chronic obstructive pulmonary disease } \\
\text { Mean age (SD) in years: TX=69.0 }(5.5) \text {; CON }=70.4 \\
(4.6)\end{array}$ & $\begin{array}{l}\text { Intervention design/setting: 'pulmonary exercises' were } \\
\text { conducted in the 'rehabilitation room', } 5000 \text { steps per day were to } \\
\text { be completed independently (not facility-based) } \\
\text { F: recommendation of } 5000 \text { steps everyday (F of 'pulmonary } \\
\text { exercises' NR); I: NR; L: } \sim 2 \text { weeks } \\
\text { Exercise specifics: 'pulmonary exercises' were conducted for } 30 \\
\text { minutes plus } 5000 \text { steps daily } \\
\text { Ancillary intervention components [treatment arm (s) only]: } \\
\text { extensive breathing exercises }\end{array}$ \\
\hline
\end{tabular}

AET, aerobic exercise training; CON, control/usual care group; D, duration of exercise session; F, frequency of exercise (days or sessions per week); I, intensity; L, length of exercise programme; NR, not reported, RCT, randomised controlled trial; RM, repetition maximum; reps, repetitions; RET, resistance exercise training; RPE, rating of perceived exertion; SD, standard deviation; TX, treatment/exercise group; $\mathrm{VO}_{2}$, oxygen consumption.

prior to surgery $[6,13,16-21,24-28,30-33]$. From baseline to the immediate pre-operative time point (mean duration 6 weeks), physical function improved significantly in prehabilitation participants compared with control participants in four studies $[16,17,19,33]$. Six studies reported significant within-group improvements in physical or psychosocial outcomes for prehabilitation participants only $(P \leq 0.05)$ $[6,13,18,20,21,24,32]$, and four studies demonstrated a worsening in physical symptoms for control subjects during the pre-operative period $[14,19,24,32]$. There were no reported between-group differences in physical or psychosocial outcomes pre-operatively in five trials $[15,18,20,30,32]$. 


\section{Postoperative outcomes}

A summary of postoperative outcomes is provided in Table A (see online supplementary material). The major findings are highlighted by category of outcome below.

HRQOL. Nine studies examined postoperative HRQOL $[14,15,20,21,23,25,28,31,33]$. Four of these studies used the Short Form Health Survey (SF-36) [14,23,28,33]. Only Back et al. [14] found between-group improvements for the prehabilitation group in any domain of HRQOL postoperatively (significantly greater emotional and physical role functioning in treatment subjects at 1 week and 6 months after surgery, respectively; $P \leq 0.05$ ) [14]. There were no significant between-group differences in HRQOL measures in seven studies $[15,20,23,25,28,31,33]$. The median risk of bias score for studies with HRQOL data was 3 (unclear risk of bias). Only one study had a low risk of bias (score of 5 or 6 ) and found no significant differences between the control and intervention groups [31].

Pain. Six studies examined postoperative pain $[15,20,23,24,31,33]$. Pain between 4 and 26 weeks postoperatively was improved in the treatment group compared with the control group in three of the five trials [20,23,33]. Only one study reported on analgesia use; Nielsen et al. found similar peri-operative and postoperative analgesia administration between study arms [20]. The median risk of bias score for studies with data on pain was 3 (unclear risk of bias). Only one study had a low risk of bias, and no significant difference between the control and intervention groups was observed [31].

Musculoskeletal and functional task performance. Sixteen of the 21 trials included a postoperative musculoskeletal or functional performance outcome [13-20,22-25,27,30-33]. Physical function was measured using physical performance tests, clinician-completed assessments (functional and questionnaire-based) and patient self-report measures. Better postoperative scores in composite physical/functional assessments for pre-operative exercisers compared with controls were observed in three trials $[16,18,30]$. D'Lima et al. found a worsening in knee function in both exercising groups compared with controls 3 weeks after surgery, although there was no difference between the groups by 48 weeks [15]. In a population of colorectal surgery patients, Carli et al. [25] found that, compared with participants who completed the prehabilitation intervention (strength training plus stationary cycling), participants in the control group (who were prescribed walking and breathing exercises) were more likely to have a clinically significant improvement in 6-minute walk test distance 2 to 4 months postoperatively $(P=0.019)$.

Functional task performance tests were improved in preoperative exercisers compared with control subjects in four of seven trials [18-20,23]. Postoperative range of motion was better in pre-operative exercisers than control subjects in four of five trials $[16,17,24,33]$. Individual performance measures of musculoskeletal fitness were improved for preoperative exercisers compared with controls in three of seven trials reporting these outcomes [14,19,33]. Three of six studies examining functional performance using self-report measures found postoperative benefits for pre-operative exercisers compared with controls [13,17,20]. No study reported a worsening of functional outcomes in the exercise group.

Results from studies with objective measures of physical function had a median risk of bias score of 3 (unclear risk of bias). Only one study had a low risk of bias [31] and found no between-group differences in a 50-m walk test.

Aerobic fitness. Postoperative aerobic fitness was measured in five trials, all using cycle ergometers to assess peak aerobic capacity $[6,13,14,21,22,25]$. Only one study demonstrated a significant improvement in postoperative aerobic fitness compared with controls [14]. In a single-arm study examining progressively intense aerobic training prior to lung resection, Jones et al. found significant decreases in peak oxygen consumption at approximately 2.5 months following lung resection despite significant improvements immediately following the intervention [6]. The median risk of bias score for studies assessing aerobic fitness was 3 (unclear risk of bias). Two studies had a risk of bias score of 4 (highest score in this outcome category); both studies found no betweengroup differences in aerobic fitness after the prehabilitation period. One study did not report postoperative changes in aerobic fitness [13], while the other study reported that insufficient participants completed the postoperative aerobic test for meaningful analysis [25].

Postoperative length of stay, healthcare utilisation and satisfaction. Eleven studies examined postoperative hospital LOS [13,15,20,22,25-29,31,32]; four of these found that patients who participated in pre-operative exercise were discharged from the hospital earlier than control patients $(P<0.01)[13,20,22,29]$, with the others showing no difference. In the trials that demonstrated reduced LOS, exercising participants were discharged 1 to 8 days (mean $=4$ days) earlier than control participants. A shorter overall LOS in the intensive care unit $(P=0.038)$ for prehabilitation participants compared with controls was also observed [13]. The median risk of bias score for studies assessing LOS was 3 (unclear risk of bias). Only one study had a low risk of bias and found no significant differences between the treatment and control groups [31].

Healthcare utilisation was assessed in three studies [13,23,32]. Arthur et al. found that a 1-day reduction in hospital LOS in the intervention group was associated with net savings of $\sim$ US $\$ 133$ per patient-day [13]. Williamson et al. reported that their pre-operative physiotherapy programme (not including the gymnasium or equipment costs) cost $£ 9$ vs $£ 15$ for pre-operative acupuncture vs no cost for home-based exercise recommendations. Discharge location was only specified in one trial, as Rooks et al. found 
that patients participating in their 6-week pre-operative exercise programme were more likely to be discharged home as opposed to a rehabilitation facility $(P<0.05)[23]$. Rodgers et al. found no difference in hospital LOS or requirement for outpatient physiotherapy between the treatment and control groups ( $P$-value not reported) [32]. Three studies assessed participant satisfaction/appreciation with the interventions $[20,26,27]$.

Peri-operative complications and adverse events. Two of the nine studies that examined the incidence of peri-operative complications found reduced rates of complications in prehabilitation patients compared with controls $[22,23]$, three trials found no difference between the intervention and control groups [26,28-30], and the other trials did not report between-group differences $[25,27,32]$. Peri-operative complication data were yielded from studies with a median risk of bias score of 2 (range $=0$ to 4 ). Study-related adverse events were reported in 14 trials $[6,13-15,17,20-23,26,27,30-32]$. Eight trials specifically reported on adverse events occurring in the various intervention arms $[6,14,17,23,26,27,30,31]$. In one trial, adverse events were attributed explicitly to exercise (drop in systolic blood pressure by $>20 \mathrm{mmHg}$ while exercising) [6,21]. The adverse event rate for exercising participants was two out of 669 participants in whole-body prehabilitation.

Meta-analysis of the postoperative length of stay outcome. Due to the heterogeneity of trial methodologies and outcome measures, it was only possible to extract sufficient data on the postoperative LOS outcome for meta-analysis. LOS data that were suitable for inclusion (i.e. compared intervention participants with controls) came from nine studies $[13,15,20,22,25-28,31]$, one of which compared two intervention arms with controls thus contributing two estimates of effect size [15]. Using the random effect model yielded a small but significant pooled Hedges' $g$ of -0.39 (95\% confidence interval -0.76 to $-0.029 ; P=0.033$ ) for the intervention group compared with the control group (Fig. 1) in a sensitivity analysis that involved removing the three studies with large standard errors in their effect size estimates (treatments 1 and 2 from D'Lima et al. [15] and Hoogeboom et al. [27]). The results showed that the pooled Hedge's $g$ was -0.54 (95\% confidence interval -0.93 to $-0.107 ; P=0.014$ ). The result remained significant.

Begg's and Egger's tests of publication bias had scores of 0.474 and 0.741 , respectively, indicating that publication bias was unlikely. However, the funnel plot indicated that there was a possible bias, with studies that had large standard errors and negative effect sizes tending to be missing (Fig. 2). The degree of heterogeneity of the 10 studies combined was high $\left(I^{2}=82.85, Q\right.$-statistic $\left.=52.47, \mathrm{df}=9 ; P=0.0001\right)$. Due to the limited number of studies, further quantitative exploration of heterogeneity was not undertaken.

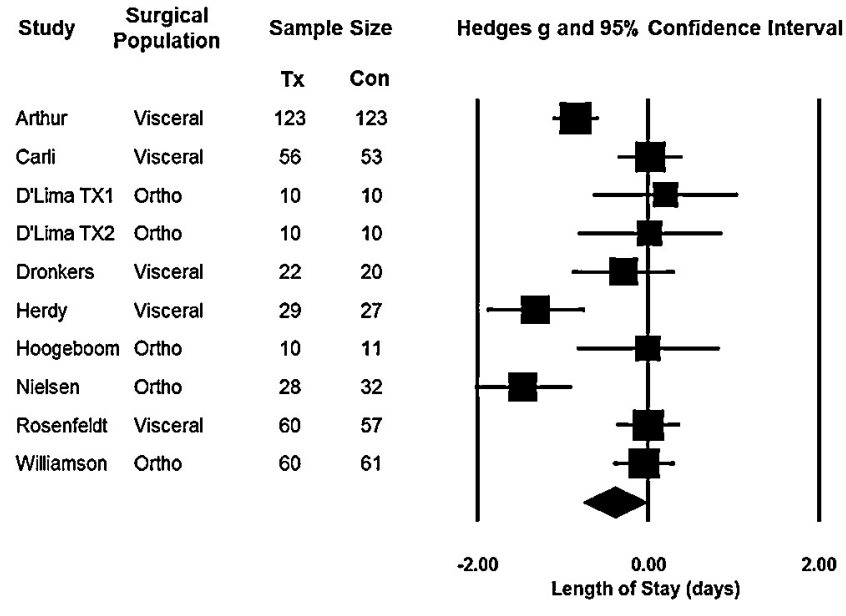

Fig. 1. Forest plot of length of stay. Ortho, orthopaedic surgery; Tx, treatment; Con, control; TX1, treatment arm 1 vs control; TX2, treatment arm 2 vs control; visceral, internal organ surgery.

\section{Discussion}

Prehabilitation has been suggested as a preventative strategy to combat pre-operative deconditioning and improve postoperative outcomes. The evidence for pre-operative totalbody prehabilitation programmes on postoperative recovery was reviewed systematically. While most of the trials included in this review had an RCT design $[6,21,32]$, the overall methodological quality was poor to moderate with an overall high to unclear risk of bias. Thus, the findings must be considered with some caution. The generalisability of these findings is further limited due to considerable prehabilitation protocol and outcome measurement heterogeneity across studies, notwithstanding the exclusion of patients with significant comorbid diseases (who may represent a population that stands to benefit most from pre-operative exercise because of greater deconditioning and vulnerability). Moreover, the study populations were primarily orthopaedic, with only

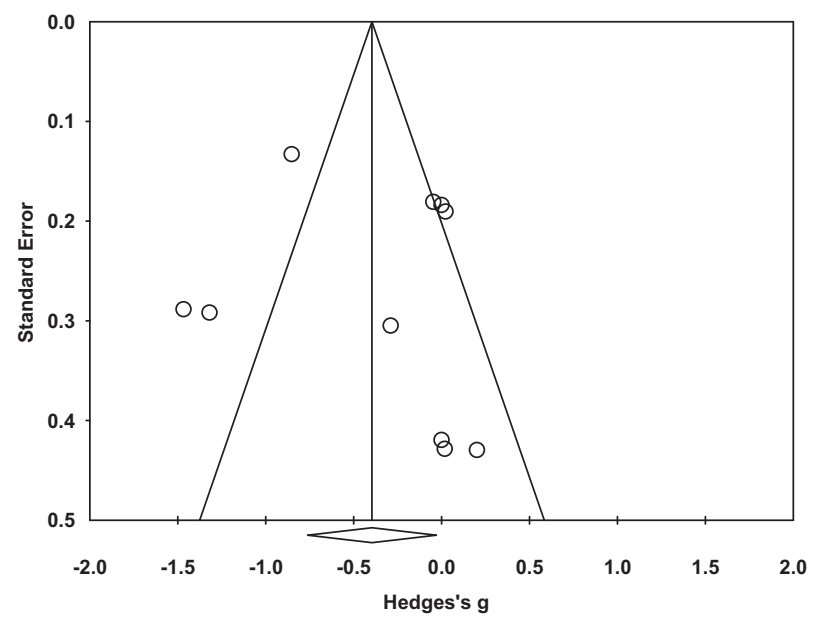

Fig. 2. Funnel plot of length of stay outcomes (standard error by Hedges' $g$ ). 
three of the 14 trials assessing lung or cardiac surgery patients $[6,13,14,21]$. There is also evidence of reporting bias, as several trials described measurement of various outcomes but did not report on those outcomes in the results [15-17,20,22,33]. These omissions in reporting tend to distort interpretation, likely overestimating the benefits of prehabilitation. Additionally, the clinical relevance of findings and reference to minimal clinically important differences in outcomes is poorly described. Only three studies powered their trials to assess clinically important differences in $\operatorname{LOS}[13,15,22]$, and only one study was powered to assess a change in a health outcome [15]. Furthermore, only four trials commented on the clinical importance of their findings [6,20-22]. Finally, although it appears that exercise is safe for patients awaiting surgery $(0.5 \%$ adverse event rate), few studies described the safety of their interventions adequately, as only four trials explicitly indicated whether the adverse events were related to the exercise intervention $[6,14,17,23]$.

This review has several limitations that should be considered. First, all non-English publications were excluded; however, the authors only found one relevant non-English study, that would probably have been excluded based on the inclusion criteria given the description of the study in the translated abstract [35]. Second, this review may be biased by the exclusion of unpublished studies, such as oral or poster presentation abstracts. However, non-peer-reviewed reports of trials typically include smaller treatment effects than formally published trials [36] and may be of poor methodological quality, potentially distorting the overall findings from the larger body of evidence. Also, the lack of consistency in outcome measurement for most performancebased or psychosocial well-being measures precluded the authors' ability to effectively meta-analyse all of the outcomes apart from LOS. Future studies should consider the pool of literature with respect to postoperative measurement for generalisability of their findings, especially in light of the well-validated measures used in many of the studies reviewed here. In this meta-analysis, significant heterogeneity was observed, indicating that the effect sizes for various outcomes were quite disparate. This may be due to the limited number of trials available for meta-analysis, as well as the variety of populations and intervention approaches that were assessed.

At present, there is no clear evidence of benefit of total-body prehabilitation with respect to postoperative functionality or psychosocial outcomes. However, the qualitative and quantitative evidence for reduced LOS, particularly in cardiac surgery patients, is more compelling, as three well-designed and adequately powered trials demonstrated reduced LOS for prehabilitation patients compared with controls [13,20,22]. The results of this meta-analysis, suggesting a reduction in LOS with prehabilitation, are intriguing and suggest the need for further high-quality studies to confirm the findings. However, the study with the lowest risk of bias found no difference in LOS between the intervention and control participants. Consequently, while a reduction in prehabilitation-related reduction in LOS would be an important clinical finding given its positive association with re-admission rates, poor functional status and mortality, and negative association with healthcare costs $[37,38]$, it is concluded that the overall evidence related to this outcome is equivocal. It is worth noting, however, that in the studies that observed reduced LOS [13,20,22], prehabilitation participants had better physical function and recovery [20], HRQOL [13] and peri-operative complications [22].

The salient nuance of this review compared with previous reviews [3-5] is the focus on total-body exercise rather than muscle- or joint-specific exercises. It is likely that totalbody exercise is better suited to prepare the entire body for prolonged sedentary behaviour, postural compensation and modified behaviour patterns that are common to postoperative recovery. The systemic benefits of cardiovascular conditioning can be achieved through a variety of modalities that may be non-, partial- or full-weight-bearing, and may prevent clinically relevant decreases in cardiopulmonary fitness. In patients awaiting lung resection, for example, Jones et al. [6] and Peddle et al. [21] demonstrated that an intense cycling intervention was tolerable and produced clinically important pre-operative increases in aerobic capacity that likely prevented postoperative decreases in aerobic fitness to levels below that required for functional independence. Moreover, they demonstrated an important positive correlation between aerobic fitness and HRQOL $(r=0.70, P \leq 0.036)$, suggesting that preservation of aerobic capacity is important for the maintenance of psychosocial health [21]. These findings require replication in high-quality RCTs and amongst other populations, but provide a starting point for further hypothesis testing. For orthopaedic surgery, training of the 'healthy' limbs or extremities likely improves the performance of various functional tasks that require improved contralateral strength and endurance to compensate for the operated limb. This review of several studies examining unilateral lower-extremity joint-replacement surgery indicates that the recovery of functional performance is often quicker in totalbody prehabilitation patients. Contrary to previous reviews that have shown a lack of benefit of prehabilitation among orthopaedic patients $[4,5]$, this review found that the majority of studies reported that total-body exercise was effective for a variety of functional outcomes. The authors have also extended the findings of previous reviews with a careful assessment of risk of bias that underscores the importance of future, high-quality study designs with low risk of bias to determine the effect of prehabilitation with more confidence.

It is likely that the best approach to prehabilitation is to consider both total-body conditioning and site-specific exercises; this should be examined further. An important remaining question is whether surgery should be postponed to allow for prehabilitation. Studies have demonstrated that the moderate delays in treatment for non-small-cell lung cancer patients are not associated with worsened long-term prognosis $[39,40]$, and postponing surgery for long enough to reach pre-operative fitness targets may improve postoperative risk 
profiles [6]. However, this issue has not been studied formally in RCTs or across other diseases.

\section{Conclusion}

The balance of data appears to favour total-body prehabilitation over standard care for patients undergoing surgery in ways that may result in significant cost savings through reduced LOS and peri-operative complications. The majority of trials included in this review suggest an improvement in physical function, LOS and pain following surgery compared with standard care. However, poor methodological quality and high risk of bias were common across studies, and preclude any definitive conclusions for all the outcomes examined. Given the nascence of this literature, future studies should focus on adequately powered RCT designs with consistent use of outcome measurements for enhanced generalisability across studies. Measures that are clinically relevant and consistent with the contemporary knowledge base include the 6-minute walk test for functional capacity, the SF-36 for physical and psychosocial quality of life, and LOS for trajectory of recovery.

\section{Funding}

DSM is funded by the Princess Margaret Foundation via the Prostate Centre for post-doctoral research, not specific to this study. There was no additional funding for this research.

\section{Conflict of interest}

None declared.

\section{Acknowledgements}

The authors would like to acknowledge the contributions of Esther Atkinson (Medical Information Specialist) for her assistance with the systematic search of databases, Dr. Crissa Guglietti for her editorial assistance, and Dr. Joseph Beyene for his statistical analysis guidance.

\section{References}

[1] Carli F, Mayo N. Measuring the outcome of surgical procedures: what are the challenges? Br J Anaesth 2001;87:531-3.
[2] Carli F, Zavorsky GS. Optimizing functional exercise capacity in the elderly surgical population. Curr Opin Clin Nutr Metab Care 2005;8:23-32.

[3] Lemanu D, Singh P, MacCormick A, Arroll B, Hill A. Effect of preoperative exercise on cardiorespiratory function and recovery after surgery: a systematic review. World J Surg 2013;37:711-20.

[4] Valkenet K, van de Port IG, Dronkers JJ, de Vries WR, Lindeman E, Backx FJ. The effects of preoperative exercise therapy on postoperative outcome: a systematic review. Clin Rehabil 2011;25:99-111.

[5] Ackerman IN, Bennell KL. Does pre-operative physiotherapy improve outcomes from lower limb joint replacement surgery? A systematic review. Austral J Physiother 2004;50:25-30.

[6] Jones LW, Peddle CJ, Eves ND, Haykowsky MJ, Courneya KS, Mackey $\mathrm{JR}$, et al. Effects of presurgical exercise training on cardiorespiratory fitness among patients undergoing thoracic surgery for malignant lung lesions. Cancer 2007;110:590-8.

[7] Santa Mina D, Matthew A, Trachtenberg J, Tomlinson G, Guglietti CL, Alibhai SMH, et al. Physical activity and quality of life after radical prostatectomy. Can Urol Assoc J 2010;4:6.

[8] Higgins JPT, Green S, editors. Cochrane handbook for systematic reviews of interventions, version 5.1.0 [updated March 2011]. The Cochrane Collaboration; 2011. Available at: www.cochranehandbook.org

[9] Comprehensive Meta-Analysis, 2nd ed. Biostat. New Jersey; 2006.

[10] Huedo-Medina TB, Sanchez-Meca J, Marin-Martinez F, Botella J. Assessing heterogeneity in meta-analysis: $Q$ statistic or $I^{2}$ index? Psychol Meth 2006;11:193-206.

[11] Begg CB, Mazumdar M. Operating characteristics of a rank correlation test for publication bias. Biometrics 1994;50:1088-101.

[12] Egger M, Davey Smith G, Schneider M, Minder C. Bias in meta-analysis detected by a simple, graphical test. Br Med J 1997;315:629-34.

[13] Arthur HM, Daniels C, McKelvie R, Hirsh J, Rush B. Effect of a preoperative intervention on preoperative and postoperative outcomes in low-risk patients awaiting elective coronary artery bypass graft surgery. A randomized, controlled trial. Ann Intern Med 2000;133:253-62.

[14] Back M, Wennerblom B, Wittboldt S, Cider A. Effects of high frequency exercise in patients before and after elective percutaneous coronary intervention. Eur J Cardiovasc Nurs 2008;7:307-13.

[15] D'Lima DD, Colwell Jr CW, Morris BA, Hardwick ME, Kozin F. The effect of preoperative exercise on total knee replacement outcomes. Clin Orthop Relat Res 1996;326:174-82.

[16] Evgeniadis G, Beneka A, Malliou P, Mavromoustakos S, Godolias G. Effects of pre- or postoperative therapeutic exercise on the quality of life, before and after total knee arthroplasty for osteoarthritis. J Back Musculoskel Rehabil 2008;21:161-9.

[17] Gilbey HJ, Ackland TR, Wang AW, Morton AR, Trouchet T, Tapper J. Exercise improves early functional recovery after total hip arthroplasty. Clin Orthop Relat Res 2003;408:193-200.

[18] Gocen Z, Sen A, Unver B, Karatosun V, Gunal I. The effect of preoperative physiotherapy and education on the outcome of total hip replacement: a prospective randomized controlled trial. Clin Rehabil 2004; 18:353-8.

[19] Jaggers JR, Simpson CD, Frost KL, Quesada PM, Topp RV, Swank AM, et al. Prehabilitation before knee arthroplasty increases postsurgical function: a case study. J Strength Cond Res 2007;21:632-4.

[20] Nielsen PR, Jorgensen LD, Dahl B, Pedersen T, Tonnesen H. Prehabilitation and early rehabilitation after spinal surgery: randomized clinical trial. Clin Rehabil 2010;24:137-48.

[21] Peddle CJ, Jones LW, Eves ND, Reiman T, Sellar CM, Winton T, et al. Effects of presurgical exercise training on quality of life in patients undergoing lung resection for suspected malignancy: a pilot study. Cancer Nurs 2009;32:158-65.

[22] Herdy AH, Marcchi PL, Vila A, Tavares C, Collaco J, Niebauer J, et al. Pre- and postoperative cardiopulmonary rehabilitation in hospitalized patients undergoing coronary artery bypass surgery: a randomized controlled trial. Am J Phys Med Rehabil 2008;87:714-9. 
[23] Rooks DS, Huang J, Bierbaum BE, Bolus SA, Rubano J, Connolly CE, et al. Effect of preoperative exercise on measures of functional status in men and women undergoing total hip and knee arthroplasty. Arthritis Rheum 2006;55:700-8.

[24] Topp R, Swank AM, Quesada PM, Nyland J, Malkani A. The effect of prehabilitation exercise on strength and functioning after total knee arthroplasty. Pm R 2009;1:729-35.

[25] Carli F, Charlebois P, Stein B, Feldman L, Zavorsky G, Kim DJ, et al. Randomized clinical trial of prehabilitation in colorectal surgery. Br J Surg 2010;97:1187-97.

[26] Dronkers JJ, Lamberts H, Reutelingsperger IM, Naber RH, DronkersLandman CM, Veldman A, et al. Preoperative therapeutic programme for elderly patients scheduled for elective abdominal oncological surgery: a randomized controlled pilot study. Clin Rehabil 2010;24:614-22.

[27] Hoogeboom TJ, Dronkers JJ, van den Ende CH, Oosting E, van Meeteren NL. Preoperative therapeutic exercise in frail elderly scheduled for total hip replacement: a randomized pilot trial. Clin Rehabil 2010;24:901-10

[28] Rosenfeldt F, Braun L, Spitzer O, Bradley S, Shepherd J, Bailey M, et al. Physical conditioning and mental stress reduction - a randomised trial in patients undergoing cardiac surgery. BMC Complement Altern Med 2011;11:20

[29] Sekine Y, Chiyo M, Iwata T, Yasufuku K, Furukawa S, Amada Y, et al. Perioperative rehabilitation and physiotherapy for lung cancer patients with chronic obstructive pulmonary disease. Jpn J Thorac Cardiovas Surg 2005;53:237-43.

[30] Wang AW, Gilbey HJ, Ackland TR. Perioperative exercise programs improve early return of ambulatory function after total hip arthroplasty: a randomized, controlled trial. Am J Phys Med Rehabil 2002;81:801-6.

[31] Williamson L, Wyatt MR, Yein K, Melton JT. Severe knee osteoarthritis: a randomized controlled trial of acupuncture, physiotherapy (supervised exercise) and standard management for patients awaiting knee replacement. Rheumatology 2007;46:1445-9.
[32] Rodgers JA, Garvin KL, Walker CW, Morford D, Urban J, Bedard J. Preoperative physical therapy in primary total knee arthroplasty. J Arthroplasty 1998;13:414-21.

[33] Ferrara PE, Rabini A, Aprile I, Maggi L, Piazzini DB, Logroscino $\mathrm{G}$, et al. Effect of pre-operative physiotherapy in patients with end-stage osteoarthritis undergoing hip arthroplasty. Clin Rehabil 2008;22:977-86 [corrected] [published erratum appears in Clin Rehabil 2008;22:1137].

[34] Gilbey HJ, Ackland TR, Tapper J, Wang AW. Perioperative exercise improves function following total hip arthroplasty: a randomized controlled trial. J Musculoskel Res 2003;7:111-23.

[35] Ródenas-Martínez S, Santos-Andrés JF, Abril-Boren C, UsabiagaBernal T, Abouh-Lais S, Aguilar-Naranjo JJ. Effectiveness of a pre-surgery rehabilitation program in total knee arthroplasty. Rehabilitacion 2008;42:4-12 [in Spanish].

[36] Hopewell S, McDonald S, Clarke MJ, Egger M. Grey literature in meta-analyses of randomized trials of health care interventions. Cochrane Database Syst Rev 2007;(2):MR000010, http://dx.doi.org/10.1002/14651858.

[37] Debes JD, Roberts RO, Jacobson DJ, Girman CJ, Lieber MM, Tindall DJ, et al. Inverse association between prostate cancer and the use of calcium channel blockers. Cancer Epidemiol Biomarkers Prev 2004; 13:255-9.

[38] Silveira JM, Winstead-Fry P. The needs of patients with cancer and their caregivers in rural areas. Oncol Nurs Forum 1997;24:71-6.

[39] Bolliger CT, Wyser C, Roser H, Soler M, Perruchoud AP. Lung scanning and exercise testing for the prediction of postoperative performance in lung resection candidates at increased risk for complications. Chest 1995;108:341-8.

[40] Jones LW, Eves ND, Peddle CJ, Courneya KS, Haykowsky M, Kumar V, et al. Effects of presurgical exercise training on systemic inflammatory markers among patients with malignant lung lesions. Appl Physiol Nutr Metab 2009;34:197-202. 